\title{
High precision flux measurements in conventional neutrino beams: the ENUBET project
}

\section{A. Longhin*}

INFN (Istituto Nazionale di Fisica Nucleare), Sezione di Padova,

via Marzolo 8, 35131, Padova (IT)

E-mail: andrea.longhinepd.infn.it

\begin{abstract}
The challenges of precision neutrino physics require measurements of absolute neutrino cross sections at the $\mathrm{GeV}$ scale with exquisite (1\%) precision. This precision is presently limited to by the uncertainties on neutrino flux at the source. A reduction of this uncertainty by one order of magnitude can be achieved monitoring the positron production in the decay tunnel originating from the $K_{e 3}$ decays of charged kaons in a sign and momentum selected narrow band beam. This novel technique enables the measurement of the most relevant cross-sections for CP violation $\left(v_{e}\right.$ and $\bar{v}_{e}$ ) with a precision of $1 \%$ and requires a special instrumented beam-line. Such non-conventional beam-line will be developed in the framework of the ENUBET Horizon-2020 Consolidator Grant, recently approved by the European Research Council. We present the Project, the first experimental results on ultra-compact calorimeters that can embedded in the instrumented decay tunnel and the advances on the simulation of the beamline. A rich program of detector $R \& D$ and acceleratorrelated activities are planned in 2016-2021.
\end{abstract}

Neutrino Oscillation Workshop

4 - 11 September, 2016

Otranto (Lecce, Italy)

* on behalf of the ENUBET Collaboration 


\section{Conventional and monitored neutrino beams}

In conventional beams the prediction of the neutrino flux is based on a full simulation of the beamline considering proton-target interactions, the reinteraction of secondaries, their tracking and decay. The simulation is constrained by beam monitoring devices and ancillary measurements (proton intensity, horn currents, beam-target misalignment etc.) while dedicated hadro-production experiments provide the particle yields from the target. In spite of these constraints, the flux prediction remains heavily dependent on ab-initio simulations leading to large systematics ( $\mathscr{O}(7-10 \%)$ ). In conventional beams the decay tunnels are almost completely passive regions.

A very precise measurement of the $v_{e}\left(\bar{v}_{e}\right)$ flux can be achieved by directly monitoring the production of $e^{+(-)}$in the decay tunnel from $K_{e 3}$ decays $\left(K^{+(-)} \rightarrow \pi^{0} e^{+(-)} v_{e}\left(\bar{v}_{e}\right)\right)$ in a sign and momentum-selected narrow band beam [1]. The positron rate is a direct handle to measure the flux of $v_{e}$ since these quantities are directly connected through the $K$ decay kinematics and the geometries of the neutrino and positron detectors. The positron rate is monitored in real time but, unlike "tagged $v$ beams" proposed since the 60's, leptons are not associated to the observed $v$ on an event-by-event basis using timing coincidences. The required time resolutions for this application are of $\sim 10$ ns thus well within reach of current technologies.

\section{The ENUBET project}

The positron monitoring approach will be tested in a conclusive manner by ENUBET (Enhanced NeUtrino BEams from kaon Tagging) [2]. The project is aimed at the design and construction of a detector capable of performing positron identification in $K_{e 3}$ decays, while operating in the harsh environment of a $v$ beam decay tunnel. The project will address all accelerator challenges of kaon tagged beams and study the precise layout of the $K / \pi$ focusing and transport system. ENUBET has been approved by the ERC (Consolidator Grant, P.I. A. Longhin) for a 5 y duration (since 1 June 2016) and a 2.0 MEUR budget. The ENUBET technology is very well suited for short baseline experiments and might enable a new generation of $v$ cross section experiments where the $v_{e}$ source could be controlled at the $1 \%$ level. It could also be exploited for sterile- $v$ experiments, especially if present anomalies would be soon confirmed. In addition, ENUBET is an important step towards a "time-tagged $v$ beam" where $v_{e}$ interactions could be time correlated with an $e^{+}$in the decay tunnel. In the ENUBET reference design (see Fig. 1, left), the positron tagger is a hollow cylinder surrounding a fraction of the decay tunnel. It is composed of a calorimetric section for $\mathrm{e}^{+}$

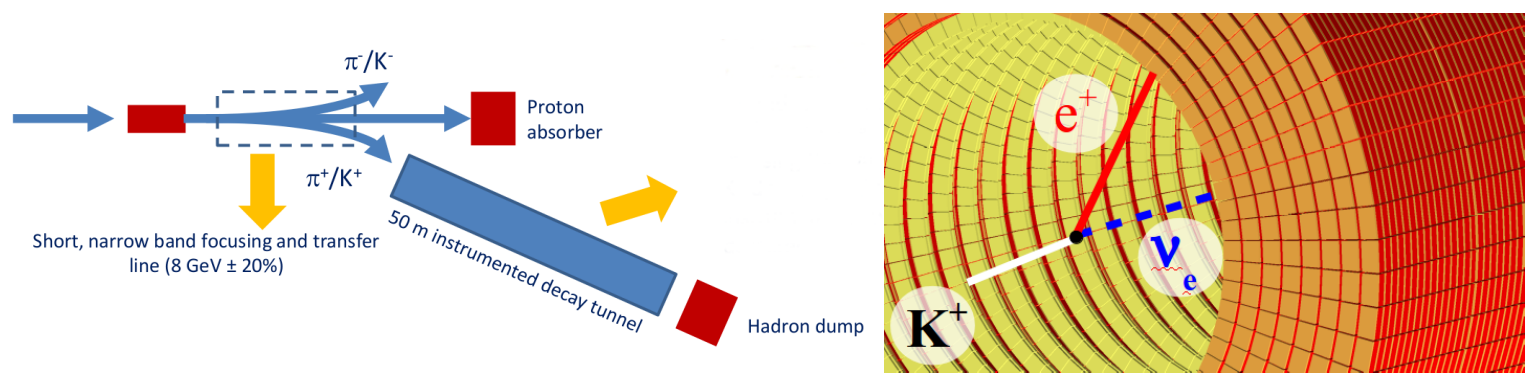

Figure 1: (Left) Layout of the beamline. (Right) A section of the positron tagger. The UCM modules are shown in red and the photon veto detector in yellow. 
tagging and $\pi^{ \pm}$rejection (in red) and a system of light tracking devices in the innermost region to reject showers from $\gamma$ conversions (photon veto, yellow). The secondary beam is designed to have an average momentum of $8.5 \mathrm{GeV}$ and $\mathrm{a} \pm 20 \%$ momentum bite. The momentum of the hadron beam and the length of the decay tunnel are chosen to:

1. reduce the fraction of $K$ decaying in the transfer line;

2. allow for $e^{+}$identification though purely calorimetric techniques;

3. match the $v$ spectrum of interest for future long baseline experiments;

4. maximize the $v_{e}$ flux from $K$ and reduce the component of $v_{e}$ from $\mu$ decays;

5. allow for a small emittance of the entering beam (few mrad over $10 \times 10 \mathrm{~cm}^{2}$ ) to prevent undecayed secondaries or $\mu$ from $\pi$ decay from hitting the tagger.

Employing a $500 \mathrm{t} v$ detector (e.g. ICARUS at Fermilab or ProtoDune-SP/DP at CERN) located $100 \mathrm{~m}$ from the entrance of the decay tunnel and a $30 \mathrm{GeV}(450 \mathrm{GeV})$ proton driver, $5 \times 10^{20}\left(5 \times 10^{19}\right)$ protons on target would allow to have a sample of $10^{4}$ tagged $v_{e}^{C C}$ interactions.

The positron tagger can be safely operated in terms of pile-up, if local particle rates are below $\sim 1 \mathrm{MHz} / \mathrm{cm}^{2}$. This can be achieved with multi-Hz slow extractions with a $\mathscr{O}(\mathrm{ms})$ duration. Extractions significantly longer than $10 \mathrm{~ms}$ are disfavored if focusing of secondaries is achieved by magnetic horns. This constraint could be removed by designing a very efficient focusing system based on DC operated magnets. Within ENUBET, proton extraction schemes compatible with accelerators at CERN, Fermilab and J-PARC will be investigated.

\section{Development and tests of the positron tagger prototypes}

The basic calorimetric unit (Ultra-Compact Module - UCM) is made of five, 15-mm thick, iron layers interleaved by 5 -mm thick plastic scintillator tiles. The transverse dimension is $3 \times 3$ $\mathrm{cm}^{2}$ for a $10 \mathrm{~cm}$ length $\left(4.3 X_{0}\right)$. Nine wavelength shifting (WLS) fibers crossing the UCM are connected directly to $1 \mathrm{~mm}^{2} \mathrm{SiPM}$ through a plastic holder (Fig. 2, left). Unlike conventional shashlik calorimeters this scheme avoids the occurrence of large passive regions usually needed to bundle the fibers and route them to a common photo-sensor. SiPMs are hosted on a PCB (Fig. 2, right) and the output signals are summed and routed to the FE electronics (fast digitizers) by Copper-Kapton lines. This readout offers outstanding flexibility in terms of choice of granularity and homegeneity in the longitudinal shower sampling.

UCM modules have been tested with cosmic rays in spring 2016 and characterized with charged particles $(e / \pi)$ in the $1-5 \mathrm{GeV}$ range at the CERN-PS East Area facility in July and November 2016 [3]. An multivariate analysis based on a Geant4 simulation confirms that the UCM $e^{+}$ identification and $\pi^{ \pm, 0}$ rejection is appropriate for ENUBET: an efficiency of $49 \%$ for $e^{+}$is obtained with $\pi^{ \pm}$and $\pi^{0}$ mis-identification probabilities of $2.9 \%$ and $1.2 \%$, respectively. During the November 2016 CERN test beam a module composed of 56 UCM in 7 longitudinal layers $\left(\sim 30 X_{0}\right)$ and of an outer module acting as an energy catcher, were exposed to $e^{-} / \pi^{-}$with grazing incidence at various incidence angles. These data, which are currently being analysed, will allow to test the 


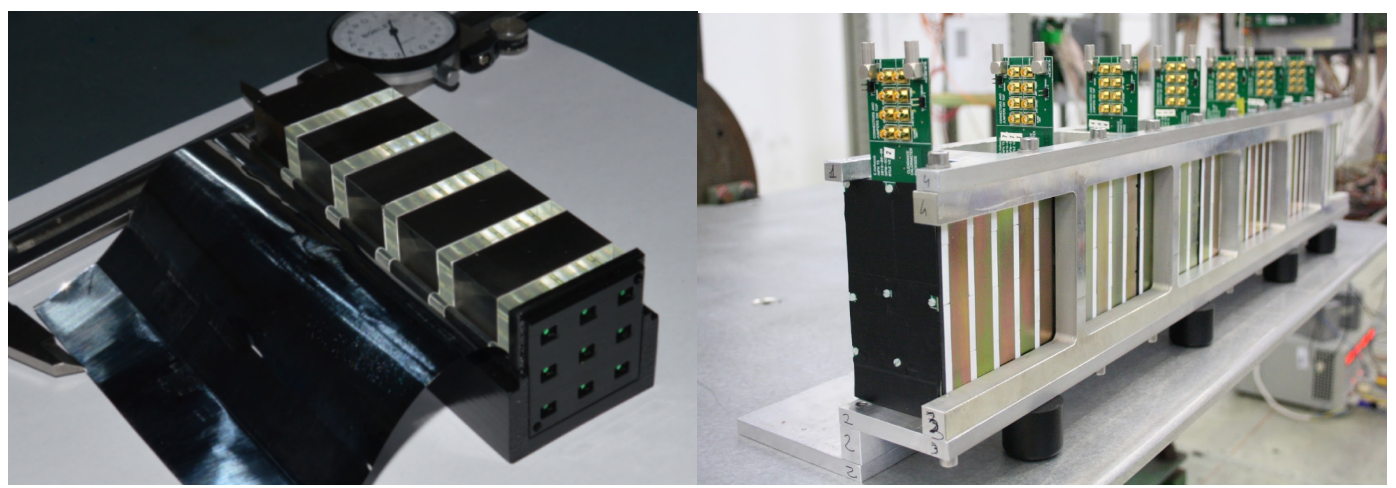

Figure 2: (Left) A single UCM. (Right) An array of 56 UCM units under test at the CERN-PS T9 area in November 2016. The PCBs holding the SiPM arrays are also visible.

expected $e / \pi^{ \pm}$performance with data under realistic conditions. In 2017 new prototypes will be tested to assess the recovery time and radiation hardness requirements. A campaign of measurements is also foreseen at the INFN-LNF BTF and at INFN-LNL to test the response to low-energy electrons/photons and neutrons respectively. Finally a full demonstrator $\left(3 \mathrm{~m}\right.$ in length, $180^{\circ}$ coverage) including the photon veto (Fig. 1, right) for the identification of $\gamma$ originating from $\pi^{0}$ will be assembled and tested at CERN.

\section{Conclusions}

The final goal of ENUBET (2016-2021) is to demonstrate that $e^{+}$-monitored $v_{e}$ beams can be built using existing technologies and hosted at CERN, Fermilab or J-PARC for a new generation of experiments on neutrino cross sections. The results obtained in the preparatory and initial phase of the project are very encouraging. Full simulations support the viability and effectiveness of the calorimetric approach whereas prototypes tests in 2016 demonstrate that shashlik calorimeters with longitudinal segmentation can fulfill the requests for $e^{+}$monitoring in the relevant energy region of few $\mathrm{GeV}$.

\section{Acknowledgments}

This project has received funding from the European Research Council (ERC) under the European Union Horizon 2020 research and innovation programme (grant agreement No 681647).

\section{References}

[1] A. Longhin, L. Ludovici and F. Terranova, Eur. Phys. J. C 75, 155 (2015).

[2] A. Berra et al. [ENUBET Coll.], CERN-SPSC-2016-036; SPSC-EOI-014.

[3] A. Berra et al., Nucl. Instrum. Methods A 830, 345 (2016).

[4] A. Meregaglia et al., Talk at IPRD16, 3-6 Oct. 2016, Siena. Italy.

[5] C. Brizzolari et al., Talk at IEEE (NSS/MIC), 29 October - 5 November 2016, Strasbourg, France. 